\title{
Huellas del contacto histórico castellano-siciliano
}

\author{
Salvatore BARTOlotTA* \\ Traces of the Castilian-Sicilian historic contact
}

\begin{abstract}
RESUMEN
Desde 1415 hasta más allá de la paz de Utrecht (1713) el castellano sustituye en Sicilia al catalán, del que no parece que queden huellas. A pesar de la aparente continuidad en la sustitución, la vida del español en la isla y su influencia en el lenguaje local, no son completamente análogas a las del catalán. El bilingüismo se difunde relativamente, pero en modo sectorial: los bilingües son a menudo solamente pasivos y cuando son activos usan el español en dominios precisos (política, administración, vida militar y sobre todo marinera, vida de corte y por lo tanto moda, danza, equitación y juegos) y en situaciones que necesiten la intervención de, al menos, un interlocutor español.
\end{abstract}

PALABRAS CLAVE: Bilingüismo / castellano / interlingüística / siciliano / sociolingüística

\section{ABSTRACT}

Since 1415 until the peace of Utrecht (1713) the Castilian substitutes the Catalan in Sicily. It seems that there are not traces left by the Catalan. In spite of the apparent continuity in the replacement, the life of Spaniards in the island, and their influence in the local language, is not completely similar to that of the Catalan. The bilingualism is relatively spread, but in a partial way: bilingual people are often only passive and when they become active, they use Spanish in specific domains (politics, administration, military life - above all marine-, court life and therefore fashion, dance, horseback riding and games) and in situations that need the participation of, at least, a Spanish speaker.

\section{KEYWORDS:}

Bilingualism / Castilian / interlinguistics / Sicilian / sociolinguistics

* UNED. Edificio de Humanidades. Depto. Filologías extranjeras y sus lingüísticas. Senda del Rey, 7. 28040 - Madrid. e-mail: sbartolotta@flog.uned.es 
Podemos afirmar con bastante exactitud que la fecha de la introducción del castellano en la isla de Sicilia fue el 2 de julio de 1415. Es posible que hubieran estado con anterioridad algunos castellanos, por no hablar de los aragoneses, de los cuales no se conocen huellas en la isla de su dialecto; pero ese día «la muy alta señora la reina doña Blanca de Navarra», vicaria de Sicilia, escribió desde Trapani una larga carta en lengua castellana «al almirante de Castilla su tío, de lo que ha de fazer en Sesilla después que la dicha señora será partida» (Starrabba 18871888: 243).

La reina Blanca era navarra, y, por lo tanto, es probable que hablara su propio dialecto; pero en Sicilia su cancillería había usado el siciliano, con rarísimas excepciones catalanas. No obstante, como el compromiso de Caspe le había entregado la corona de Aragón, y, por ende, la de Sicilia, a Fernando el Trastámara, de familia castellana, y el destinatario de la carta era Alfonso Enríquez, almirante de Castilla, que había acompañado al nuevo virrey de la isla, el infante Juan de Peñafiel, la vicaria, que estaba a punto de abandonar su cargo, consideró necesario dirigir al magnate castellano un documento en su propia lengua, aunque impregnada de bastantes catalanismos. Fue un error: la era catalana en Sicilia no se había terminado. La corte aragonesa de Nápoles, al menos nominalmente, era cuadrilingüe (latín, italiano, catalán y castellano) y, a pesar de que algunos virreyes del siglo XV fueran castellanos, el uso del castellano en Sicilia era una excepción a finales del siglo XV, no percibiéndose en el lenguaje la unión de las coronas de Aragón y Castilla y su repercusión en el imperio español en Italia, en el cuadro de una política de predominio en la península.

Desde 1500 hasta más allá de la paz de Utrecht de 1713, el español sustituye en Sicilia al catalán, del que no parece que queden huellas. A pesar de la aparente continuidad en la sustitución, la vida del español en la isla y su influencia en el lenguaje local no son completamente análogas a las del catalán. Los grupos en los que surge el contacto lingüístico son a primera vista los mismos. En primer lugar, los rangos políticos y administrativos: casi siempre españoles son los virreyes y otros titulares de altos cargos civiles y religiosos (los religiosos son seguramente más numerosos de lo que lo fueron los catalanes, y no se puede olvidar la presencia española a través de la Inquisición); sin dejar de lado los altos cargos militares, lo que no había pasado antes, existía, sin duda alguna, la nobleza catalana o aragonesa, pero los nobles llegaban a la isla, en gran parte, para quedarse, asimilando la cultura y la lengua del lugar; ahora en cambio se trataba de funcionarios, dotados de alto prestigio social, pero mucho más a menudo en una etapa transitoria, dentro de una carrera que los llevaba de un lugar a otro del inmenso imperio. Esta presencia transitoria y común a españoles de todos los niveles sociales, del virrey al soldado, hace que el contacto sea bastante importante desde el punto de vista cuantitativo, pero más fiel al prestigio que a la vida en común. El español se habla en la corte, en los palacios episcopales o inquisitoriales, en los cuarteles, y posee una valoración social extremadamente positiva, detrás de la que se encuentran el imperio, la cultura y la moda; pero el contacto con los locales es menos 
íntimo de lo que se podría pensar, sea porque no disminuye una tenaz corriente de recíproca falta de estima (por no decir antipatía) sea porque faltan grupos más dinámicos, los que en el caso catalán habían sido los mercaderes, los importadores y los exportadores.

La marca y el efecto de este tipo particular de contacto, más cercano a condiciones propiamente coloniales de lo que hubiera sido el sículo-catalán, y en el altísimo número de préstamos del español hace que la lengua hablada se invada de castellanismos; pero es raro que una palabra española enraíce en el dialecto vivo. El bilingüismo se difunde relativamente, pero de modo sectorial: los bilingües son a menudo solamente pasivos y cuando son activos usan el español en dominios concretos (la política, administración, vida militar y, sobre todo, marinera, vida de corte y, por lo tanto, moda, danza, equitación y juegos) y en situaciones que necesiten la intervención de al menos un interlocutor español. Sobre la prevalencia del dominio pasivo es interesante este avvertimento de Argisto Giuffredi:

Dove sopra trattai de' libri che dovrete leggere, mi scordai dirvi che leggeste libri spagnuoli per intender la linga; né ve ne fate beffe, poiché a tutti i vassalli del Re Nostro Signore ci conviene, se non parlarla, almeno intenderla per mille buoni rispetti: ben consiglio che innanzi un superiore nessuno la parli, se non la sa bene. Pero intenderla ci è necessario ${ }^{1}$.

Nótese la formalidad de las situaciones en que el hablante siciliano podrá usar el español. Bastante limitado aparece, por otro lado, el uso escrito: según resulta del análisis (Vàrvaro 1977: 5-7) de unos trescientos documentos de los años 1670-1680, la correspondencia entre la corte de Madrid y la virreinal se hacía en español y se responde en la misma lengua. Sin embargo, los súbditos sicilianos escriben en italiano, lengua de normal uso escrito de todos los que desempeñan cargos públicos civiles en la isla, sin tener en cuenta su origen. Hasta en el ejército, cuya lengua oficial era el español, se usa el italiano si quien escribe es italiano o, español si se dirige a los civiles. Según lo dicho anteriormente, queda claro que, desde el punto de vista oficial, el español (lengua del Rey Nuestro Señor) resalta y no tiene derecho a ser sustituido por el italiano (lengua escrita del reino). Aunque normalmente como quien escribe es de origen ibérico, resulta un producto extremadamente híbrido, como el acta del proceso del tribunal de la Inquisición, escrita en Messina en 1555:

Coram Illustre et R.mo domino don Bartholomeo Sebastián, episcopo Pactensi et Inquisitore, comparuit quedam muger que, medio juramento, interrogata dixit che si chiama soro Elisabeta d'Afannato, monaca di lo terço ordine di l'ordini di li Escapuchini, et primo ffo maritata con Jacobo l'Afannato, che campaba di rendita et

1 El párrafo de los Avvertimenti cristiani de Argisto Giuffredi es citado por Sorrento (1921: 98-99). «Donde había tratado anteriormente de los libros que tienen que leer, se me olvidó decirles que leyeran libros españoles para entender la lengua; no se burlen, porque a todos los vasallos del Rey Nuestro Señor nos conviene, si no hablarla, al menos entenderla para ser respetuosos: les aconsejo que delante de un superior nadie la hable, si no la conoce bien. Pero entenderla es necesario». 
mercanzia, et per discarrigo di sua consciencia et timore di li edicti vinni a revelari lo infrascripto, et es di etati di anni trentacinco vel circa².

Dejando de lado las fórmulas jurídicas latinas, copiadas en cursiva, no se puede decir, según Garufi, que el secretario «evidentemente un español, escribió un siciliano mixto con formas ortográficas y dialectales propias de su lengua». En realidad, está clara la intención de verbalizar en italiano una declaración hecha ciertamente en siciliano. De aquí formas como soro, di li, ordini, vinni, revelari, etati y trentacinco, pero el secretario tenía experiencia con los escritos en siciliano, si usa ch por chi en chama y si duda en cuanto a las dobles consonantes de Elisabeta y Afannato (terço puede ser un hispanismo gráfico). Por otro lado, el español no sólo determina grafías como Escapuchini (sea por la $s$ prostética que por la ch como representación de [i]], que podría ser siciliano, como el dichiano que se lee más abajo), campaba, discarrigo y consciencia, pero aparece directamente en formas como mugery es, fuera de los párrafos de verdadera verbalización.

La confusión es imprevisible, como en todos los casos de mezcla lingüística caótica, más allá de todas las normas reconocidas colectivamente. He aquí otras líneas: «portao uno core di chera, in lo quale estabano apitzati certi espinguj et pulbure nigre et li dissi al suo parere: «Ecco la maya, che l'ayo trobato»»33.

Tenemos en casos como éste, por cierto bastante frecuentes, una situación de contacto caótico, que mezcla en la página escrita rasgos gráficos, fonéticos, gramaticales y léxicos de tres sistemas diferentes, sin lograr establecer un sistema de interferencias regular y constante, que es lo que constituye la premisa para la influencia profunda de una lengua sobre otra.

Muy diferente, e igualmente significativo, es el caso de las cartas de don Pedro Paolini - tenaz defensor de Francavilla en 1677- durante el periodo de la guerra de Messina 4 . Paolini realmente no era siciliano, sino de la Italia central, pero su lengua es la misma de los muchos grados medios y bajos del ejército español en la isla. En el fondo italiano de sus cartas, no faltan fenómenos análogos a los que hemos encontrado bajo la pluma del secretario del tribunal de la Inquisición hace más de un siglo: él escribe alguna vez Francaviglia ${ }^{5}$, convirtiendo en grafía italiana la

2 Cito la edición de Carlo A. GARUFI, en Archivio Storico Siciliano, XXXIX, 1914: 354. «Coram Illustre et R.mo domino don Bartholomeo Sebastián, episcopo Pactensi et Inquisitore, comparuit quedam muger que, medio juramento, interrogata dixit que se llama sor Elisabeta de Afannato, monja terciaria capuchina, y primo se casó con Jacobo el Afannato, que vivía de renta y mercancía, y para descargar su conciencia y temor de los edictos vino a revelar la escritura, y cuenta con treinta y cinco años vel circa».

3 Ibídem, pág. 354. «Llevé un corazón de cera en el que estaban clavados algunos alfileres y un polvo negro y le dije a su parecer: «He aquí la «maya», que he encontrado»».

4 Para la bibliografía referente a la revuelta de Messina, puede consultarse, entre otros, Giacomo GALATTI (1899), Giuseppe ARENAPRIMO (1904: 70-137), Francesco GUARDIONE (1906 y 1907), Umberto DALLA VECCHIA (1907), Máximo PETROCCHI (1954), Emile LALOY (1929-1931), Saverio DI BELLA (1979) y $\left(2001^{2}\right)$ y Luis Antonio RIBOT (1982), (1991: 183-199), (1999: 121-130), (2002) y (2004: 101-128).

5 Cf. Archivio Storico Siciliano, XXIV, 1899: 154-155 y 157. 
pronunciación española de la grafía italiana del topónimo siciliano, ni faltan sicilianismos como abbrugari, tutti li casi 'las casas', mandari, la bestiame, carricasse, quarche 'algún', arbitrij 'campos cultivados', donano 'dan', praija 'playa'. mucho más interesantes son las oraciones como

(1) Hanno avuto attrevimento d'avanzarsi sotto Castiglione.

(2) Supplico a V. E. a non disempararmi in questa frontiera».

(3) Questa terra [de Moncuffi] mi à mandato ad avvisare che volentieri si averia di novo rintregata alla obbidienza.

(4) L'inemigo si trova con quarche scarsezza di pane.

(5) Per accertare maggiormente il servitio del re.

(6) Se Dio guardante succede il caso.

(7) II nemico in detto giorno del venerdi abbassò la cavalleria e pedoni nella piana di Tauormina.

(8) Con mille trampe et sotterfugij donano ad intendere alli superiori tutte quelle materie che cercano in loro beneficio [...] si sviscerano per l'accerto del real servitio.

(9) Lo tengho per assentato che marci per questa parte. ${ }^{7}$

Aquí ya no se trata de mezcla caótica: el italiano se vuelve permeable a un gran número de préstamos del español, a veces solo semánticos (como frontiera 'zona del frente' y a veces completamente gratuitos (como inemigo), que llegan a formar enteras locuciones (como Dio guardante impresa sobre Dios mediante y tenere per assentato). La permeabilidad se debe al dominio específico en que se coloca el texto, ya que todos los préstamos corresponden al lenguaje militar (attrevimento, disemparare, frontiera, rintregare, inemigo, abbassare, trampa) o burocrático (accertare, accerto, assentato). Eso revela la fuerza y, al mismo tiempo, la debilidad del influjo español: el contacto prolongado y estrecho en situaciones de ese tipo, mayor en la lengua hablada que en la escrita, como consecuencia de la presencia de un gran número de españoles, determinaba un largo flujo de préstamos; pero cuando las situaciones cambien, cuando los españoles desaparezcan de la burocracia, del ejército y de la iglesia, cuando disminuya el prestigio de la cultura y del modo de vivir español, entonces los préstamos revelarán su debilidad y finalmente desaparecerán.

Sería erróneo fechar esta desaparición en 1713, es decir, al final del gobierno virreinal. Las cartas relativas al gobierno de Carlos VI de Austria en Messina entre

\footnotetext{
${ }^{6}$ Las cartas de Paolini están entre los documentos editados y registrados por S. CHIARAMONTE, en Archivio Storico Siciliano, XXIV, 1899: 77-209 y 498-585. A esta edición se refieren los reenvíos en las citaciones que siguen.

7 Ibídem. (1) «Se han atrevido a presentarse ante Castellón» (pág. 138). (2) «Le suplico a V. E. que no me desampare en este frente» (pág. 139). (3) «Esta tierra [de Moncuffi] me ha mandado para que le avise que con gusto se habría entregado de nuevo a la obediencia» (pág. 159). (4) «El enemigo se encuentra con alguna escasez de pan» (ibíd.). (5) «Para acertar mayormente el servicio del rey» (pág. 162). (6) «Si Dios mediante pasa algo» (pág. 163). (7) «El enemigo en dicho día del viernes abajó la caballería y los soldados en la altiplanicie de Taormina» (pág. 179). (8) «Con mil trampas y engaños dan a entender a los superiores todas las cuestiones que los beneficia [...] se descabellan para acertar en el real servicio» (pág. 190). (9) «Tengo por cierto que marche por esta parte» (pág. 193).
} 
1719 y $1734^{8}$ muestran que el emperador escribe en Sicilia siempre en español y con fórmulas españolas (incluso "yo el Rey») y que se escribe desde Messina hasta Viena en la misma lengua. El gobierno de los Austrias se mantiene sin cambios en los usos de la corte española: de hecho se trataba, en ambos casos, de la misma casa de Austria y el emperador pretendía la sucesión de Madrid.

Es inútil hacer un inventario, aunque sea parcial, de los hispanismos que no perduraron, porque forman parte integral de la lengua usada en Sicilia entre los siglos XVI y XVIII'. Se podrían señalar algunos casos de términos que han adquirido una vitalidad duradera. Así, por ejemplo, attrasari 'retrasar', que originariamente es una palabra típica del ámbito burocrático, civil y militar (se usa siempre en relación con los pagos), pero acaba asumiendo caracteres de exponente de un modo de conducir la administración y entra en el habla con el sentido general de, 'retrasar' y, en el reflexivo, 'retrasarse'. Menos fácil es identificar el sector por donde pasa un verbo de sentido bastante general, como el español buscar, que en 1500 produce el italiano buscare 'obtener, procurar, ganar'10; en Sicilia vuscari se verifica ya en Palermo en 1459, en el sentido original y con el equivalente autóctono, probablemente como préstamo de ambiente judío-español «vanno buscando circando li debiti» ${ }^{11}$, pero, se encajó sólidamente con los españoles, manteniendo por mucho tiempo - al contrario de lo que pasaba en otros lugares - el valor 'buscar' en los primeros decenios del siglo XVII en Palermo: «fôro forzati di andare molt'uomini deIle terre con zappi, zapponi e pali per boscare il tabuto tra la neve ${ }^{12}$, pero acabando con especializarse en la locución vuscàrisi u pani 'ganarse el pan'; la perfecta introducción de la voz se confirma por el pasaje de $-b a-v$, fenómeno bastante raro en los préstamos tardíos.

La supervivencia hasta el siglo XIX o inicios del XX de palabras como faudigghia 'traje de paño negro de seda usado un tiempo por las mujeres, a modo de falda, acompañada por otra parte del busto y del cuello' o fadillinu 'faldita de tela de seda de colores con diseños y cintas, usada en el campo durante las festividades' (Traina 1868: 394) se ha debido, en cambio, no tanto a su perfecta introducción en la familia léxica de fauda 'la parte del traje que va de la cintura para bajo'(Traina 1868: 391), en cuanto a la supervivencia de la respectiva ropa en áreas fuertemente conservadoras. Por lo general, los términos relacionados con la moda española han sido eliminados por la evolución del gusto y han sobrevivido marginalmente con connotaciones peyorativas, como giammerga 'traje deformado y ridículo', que originariamente es un traje con dos faldas, puesto de moda por las

8 Publicadas por R. Martini, en Archivio Storico Siciliano, XIX, 1904: 34-58.

9 Basta aquí el reenvío al libro de Gian Luigi BECCARIA 1968, ejemplares como impostación general y muy útil también por lo que se refiere específicamente a Sicilia.

${ }_{10} \mathrm{Cf}$. Salvatore BATTAGLIA 1962-1998: II: 461. Nótese que el primer ejemplo deriva de las Canciones Carnavalescas, sin una fecha precisa.

11 Cf. Lagumina - Lagumina 1884-1895: II: 46. «Van buscando las deudas».

12 Cf. Di Marzo 1869-1886: II: 204. «Fueron obligados a salir de las tierras muchos hombres con azadas, azadones y palas para buscar el ataúd entre la nieve». 
tropas del mariscal Schomberg durante la guerra de Cataluña (1650 aproximadamente) y por él llamado en español chamberga (DRAE 1998: vol. 1: 634-635).

\section{REFERENCIAS BIBLIOGRÁFICAS}

AlfierI, Gabriella (1992): «La Sicilia», en Francesco BRUNI (ed.), L'italiano nelle regioni. Lingua nazionale e identità regionali, Torino: UTET, 798-860.

AmbrosinI, RICCARDo (1977): Stratigrafia lessicale di testi siciliani dei secoli XIV e XV, Palermo: Centro di Studi Filologici e Linguistici Siciliani.

ArenAPRIMO, GIUSEPPE (1904): «Gli esuli messinesi del 1678-79», Archivio Storico Messinese, 5, 70-137.

Bartolotta, Salvatore (2000): Perfil de historia lingüística de Sicilia en la edad aragonesa y castellana, trabajo de investigación, Madrid: UNED.

BARTOLOTta, Salvatore (2004): «Breve recorrido histórico-lingüístico en la Sicilia de la Guerra de las Vísperas", Espacio, Tiempo y Forma, Serie III, 17, 67-78.

Bartolotta, Salvatore (2005A): I/ dialetto di Castrofilippo, Bari: Laterza.

BARTOLOTTA, Salvatore (2005B): «Las interferencias entre el siciliano y el latín en el siglo XIV», en Jenaro COSTAS RODRÍGUEZ (ed.), Ad amicam amicissime scripta, Madrid: UNED, II, 201-208.

BARTOLOTTA, SalVATORE (2006A): «Interferencia catalano-siciliana en el Reino de Aragón», en Milka VILLAYANDRE LLAMAZARES (ed.), Actas del XXXV Simposio Internacional de la Sociedad Española de Lingüística, León, 12-15 de diciembre de 2005, Universidad de León, Dpto. Filología Hispánica y Clásica, 211-220.

BARTOLOTTA, Salvatore (2006B): «Fenómenos de interferencia en la Sicilia no románica», Interlingüística, 17, 175-182.

Bartolotta, Salvatore (2007): «Koiné siculo-toscanizada en los dominios de la corona de Aragón", Espacio, Tiempo y Forma, Serie III, 20, 315-324.

Bartolotta, Salvatore; Lunetta, S.; Messina, C.; Tedeschi, P.; y Virgillitto, S. (1993): "Confessionale II»: Analisi linguistica e confronto con «Libru di lu transitu et vita di messer Sanctu Iheronium» ed "Ordini di la confessioni Renovamini», trabajo de investigación, Catania: Università degli Studi di Catania.

Battaglia, Salvatore (1962-2002): Grande dizionario della lingua italiana, 21 vols., Torino: UTET.

BeCCARIA, GIAn LUIGI (1968): Spagnolo e spagnoli in Italia, Torino: Giappichelli.

Bresc, HenRI (1971): Livre et société en Sicile (1299-1499), Palermo: Centro di Studi Filologici e Linguistici Siciliani.

Bresc, HeNRI (1986): Un monde méditerranéen. Économie et société en Sicile. 1300-1450, Roma - Palermo: Ecole Française de Rome - Accademia di Scienze, Lettere e Arti di Palermo. 
Bresc, Henri y Goitein, Shelomo D. (1970): «Un inventaire dotal des juifs siciliens (1479)», Mélanges d'archéologie et d'histore de l'Ecole française de Rome, 82, 903-917.

BRUNI, FrANCESCO (1980): «La cultura e la prosa volgare nel '300 e nel '400», Storia della Sicilia, IV, 179-278.

BRUNI, FRANCESCO (ed.) (1992): L'italiano nelle regioni. Lingua nazionale e identità regionali, Torino: UTET.

Corrao, Pietro (1991): Governare un regno. Potere, società e istituzioni in Sicilia tra Trecento e Quattrocento, Napoli: Liguori.

D'Agostino, Guido (1979): Parlamento e società nel regno di Napoli. Secoli XV - XVII, Napoli: Guida.

D’Alessandro, Vincenzo (1963): Politica e società nella Sicilia aragonese, Palermo: Manfredi.

D’Alessandro, Vincenzo (1986): «ll Mezzogiorno dagli Angioini agli Aragonesi», La storia. I grandi problemi dal Medioevo all'Età contemporanea, II, 523-533.

DALLA VeCCHIA, UMBeRTo (1907): Cause economiche e sociali dellinsurrezione messinese del 1674, Messina: Muglia.

Del Treppo, Mario (1972): I mercanti catalani e l'espansione della corona aragonese nel secolo XV, Napoli: Collana del Seminario di Storia medioevale e moderna dell'Università di Napoli, 4.

Del Treppo, Mario (1986): «\|l regno aragonese», G. Galasso y R. Romeo (eds.), Storia del Mezzogiorno, IV, Roma - Napoli: Edizioni del Sole, 89-201.

Di Bella, SAVERIO (1979): La rivolta di Messina (1674-1678) e il mondo mediterraneo nella seconda metà del Seicento, Luigi Pellegrini editore: Cosenza, $2001^{2}$.

DRAE (2001): Diccionario de la lengua de la Real Academia Española, 2 vols., XXII ed., Madrid: Espasa Calpe.

Elliot, Jonhn H. ; Villari, Rosario ; Hespanha, Antonio Manoel ; Anatra, Bruno et alil (eds.) (1991): 1640: La monarquía hispánica en crisis, Barcelona: Crítica.

Galasso, Giuseppe (1992): I/ Regno di Napoli. Il Mezzogiorno angioino e aragonese (12661494), Torino: UTET.

Galasso, Giuseppe y Romeo, Rosario (eds.) (1986): Storia del Mezzogiorno, vol. IV, Roma Napoli: Edizioni del Sole.

Galattı, Giacomo (1899): La rivoluzione e l'assedio di Messina (1674-78), Messina: Nicotra.

GIUNTA, FRANCESCO (1973): «Un gruppo di lettere in siciliano di Eleonora d'Aragona (1375)», Bollettino del Centro di Studi Filologici e Linguistici Siciliani, 12, 346-353.

Guardione, Francesco (1906): Storia della rivoluzione di Messina contro la Spagna, 1671 1680. Documenti, Palermo: Scuola tip. «Boccone del Povero».

Guardione, Francesco (1907): Storia. La rivoluzione di Messina contro la Spagna, Palermo: Recher. 
Holtus, GüNTER (1989): «Per una carta dialettologica italiana», en Günter HOLTUS, Michael METZELTIN y Max PFISTER (eds.), La dialettologia italiana oggi. Studi offerti a Manlio Cortelazzo, Tübingen: Narr, XXXII y LIII-LVI.

Holtus, Günter; Metzeltin, Michael; y Pfister Max (eds.) (1989): La dialettologia italiana oggi. Studi offerti a Manlio Cortelazzo, Tübingen: Narr.

LALOY, EMILE (1929-31): La révolte de Messine, l'expédition de Sicile et le politique française en Italie (1674-1678), avec des chapitres sur les origines de la révolte, (1674-1678), et sur la sorte des exilés, (1678-1702), Paris: C. Klincksieck.

Leone, Alfonso (ed.) (1990): /l vocabolario siciliano - latino di Lucio Cristoforo Scobar, Palermo: Centro di Studi Filologici e Linguistici Siciliani.

LI GotTI, ETTORE (ed.) (1951): Volgare nostro siculo. Crestomazia di testi siciliani del sec. XIV, I, Firenze: La Nuova Italia.

MATTESINI, ENZO (1994): «Sicilia», en Luca SERIANNI y Pietro TRIFONE (eds.), Storia della lingua italiana, Torino: Einaudi, III, 406-432.

MigliorinI, BRUno (1988): Storia della lingua italiana, 2 vols., Firenze: Sansoni (reim. con la introducción de G. Ghinassi).

MocciARO, A. G. (1975): «Vocabolari siciliani manoscritti inediti», La ricerca dialettale, 1, 423444.

NALLI, PAOLO (1938): «Saggio bibliografico sui testi a stampa in dialetto siciliano», Scritti vari dedicati a Mario Armanni in occasione del suo sessantesimo compleanno, Milano: Hoepli, 159-198.

Pagliaro, Antonino (ed.) (1953): Saggi di critica semantica, Messina - Firenze: D’Anna.

Pagliaro, Antonino (ed.) (1956): Nuovi saggi di critica semantica, Messina - Firenze: D'Anna.

Pellegrini, Giovani Battista (1977): Carta dei dialetto d'Italia, Pisa: Pacini.

Petrocchi, Massimo (1954): La rivoluzione cittadina messinese del 1674, Firenze: Le Monnier.

PICcItTo, GIORGIO (1950): «La classificazione delle parlate siciliane e la metafonesi in Sicilia», Archivio storico per la Sicilia orientale, serie IV, III, 5-34.

PITRÈ, GIUSEPPE (1928): «Supplemento ai dizionari siciliani», Studi Glottologici Italiani, VIII, 1119.

QuAtTordio Moreschini, Adriana (ed.) (1984): Tre millenni di storia linguistica della Sicilia (Atti del Convegno della Società Italiana di Glottologia, Palermo 25-27 marzo 1983), Pisa: Giardini.

Ribot García, LUIS ANTONIO (1982): La revuelta antiespañola de Mesina. Causas y antecedentes (1591-1674), Valladolid: Universidad de Valladolid, Facultad de Filosofia y Letras.

Ribot GarcíA, LUIS ANTONIO (1991): «Las revueltas sicilianas de 1647-1648», en J. H. ELLIOT, R. VILlaRI, A. M. Hespanha, B. AnAtra et alii (eds.), 1640: La monarquía hispánica en crisis, Barcelona: Crítica, 183-199. 
Ribot García, Luis Antonio (1999): «Las revueltas Italianas de Nápoles y Sicilia (16471648)», Cuadernos de Historia Moderna, 11, 121-130.

Ribot GaRcía, Luis Antonio (2002): La monarquía de España y la guerra de Mesina (16741678), San Sebastián de los Reyes (Madrid): Editorial Actas.

RIBOt GarcíA, LUIS ANTONIO (2004): «Las revueltas Italianas del siglo XVII», Studia Historica. Historia Moderna, 26, 101-128.

RohlFS, Gerhard (1975): Historische Sprachschichten in modernen Sizilien, München: Bayerischen Akademie d. Wissenschaft (tr. it. La Sicilia nei secoli, Palermo: Sellerio, 1984).

Rohlfs, Gerhard (1977): Supplemento ai vocabolari siciliani, München: Bayerischen Akademie d. Wissenschaft.

Romano, D. (1973): «Un testo in volgare siciliano del 1351», Culture regionali e letteratura nazionale (Atti del VII Congresso dell'Associazione Internazionale per gli Studi di Lingua e Letteratura Italiana, Bari 31 marzo-4 aprile 1970), Bari: Adriatica Editrice, 387-391.

RufFINO, Gıovanni (1984): «Isoglosse siciliane», A. Quattordio Moreschini (ed.), Tre millenni di storia linguistica della Sicilia (Atti del Convegno della Società Italiana di Glottologia, Palermo 25-27 marzo 1983), Pisa: Giardini, 161-224.

Serianni, Luca y Trifone, Pietro. (eds.) (1994): Storia della lingua italiana, 3 vols., Torino: Einaudi.

Starrabba, Raffaele (1873): «Processo di fellonia contro frate Simone Del Pozzo vescovo di Catania (1392)», Archivio Storico Siciliano, I, 401-402.

StARRABBA, RAFFAELE (ed.) (1887-1888): Lettere e documenti relativi a un periodo del vicariato della Regina Bianca in Sicilia (1411-1412), Palermo: Società Italiana per la Storia Patria (reimp. 1993).

Traina, Antonino. (1868): Nuovo vocabolario siciliano - italiano, Palermo: Edizioni Sedilis (reimp. 1977).

Traina, Antonino (1877-88): Vocabolarietto delle voci siciliane dissimili dalle italiane, Palermo: Libr. Intern. L. Pedone-Lauriel di C. Clausen.

Tramontana, Salvatore (1989): Gli anni del Vespro, Bari: Dedalo.

VÀrVARO, A. (1974): «Prima ricognizione dei catalanismi nel dialetto siciliano», Medioevo romanzo, I, 86-110.

VÀrVAro, Alberto (1977): «Note per la storia degli usi linguistici in Sicilia», Lingua Nostra, XXXVIII, 1-7.

VÀrvaro, Alberto (1979): Profilo di storia linguistica della Sicilia, Palermo: Flaccovio.

VÀrvaro, Alberto (1981): Lingua e storia in Sicilia. Dalle guerre puniche alla conquista normanna, Palermo: Sellerio.

VÀrVAro, Alberto (1984): La parola nel tempo. Lingua, società e storia, Bologna: II Mulino.

VÀrVAro, Alberto (1988): «Aree linguistiche, XII. Sicilia», Lexikon der Romanischen Linguistik, 4, 716-731. 
Vitolo, Giovanni (1986): II regno angioino, en Giuseppe Galasso y Rosario Romeo (eds.), Storia del Mezzogiorno, IV, Roma - Napoli: Edizioni del Sole, 11-86.

Vitolo, Giovanni (1994): Corso di Storia I. Medioevo, Milano: Bompiani.

VS (1977-2002): Vocabolario siciliano, Vol. I (A-E), G. Piccitto (ed.), Catania - Palermo: Centro di Studi Filologici e Linguistici Siciliani, 1977; Vol. II (F-M), G. Tropea (ed.), Catania Palermo: Centro di Studi Filologici e Linguistici Siciliani, 1985; Vol. III (N-Q), G. Tropea (ed.), Catania - Palermo: Centro di Studi Filologici e Linguistici Siciliani, 1990; Vol. IV (RS), G. Tropea (ed.), Catania - Palermo: Centro di Studi Filologici e Linguistici Siciliani, 1997; Vol. V (Si-Z), S. C. Trovato (ed.), Catania - Palermo: Centro di Studi Filologici e Linguistici Siciliani, 2002. 